\title{
PROSPECTS
}

\section{Career resilience}

\section{It's not enough to be an expert on a specific topic. Today's scientists also need to be able to apply their knowledge, argues Peter Fiske.}

Some might assume that cranking out good results is all that is necessary to further a career. But such a 'career protocol' is not safe. That has never been more true than now, as the world tries to climb out of the current recession.

Although the economy has shown signs of improvement, scientists and engineers of all ages will be feeling the effects of the recession for some time. In the past, the scientific community was often insulated from the brunt of economic downturns. Buoyed by the conviction that the future relied on science and technology, many scientists and engineers may have viewed the onset of this current economic storm with some complacency.

Well, no more. Unlike previous recessions, this one has reached into academia directly through budget cuts to higher-education institutions, especially in the United States at, for example, state-funded universities. And the negative effect on research funding may persist longer than normal. Despite efforts in the United States to boost science funding via stimulus spending, federal and state governments will be left with historically large budget deficits to manage: the pressure to cut discretionary government spending will be enormous. And with big losses to retirement portfolios, many senior scientists and engineers will probably remain at their benches for a few years longer to rebuild their nest eggs.

Yes, the news is grim. But I would argue that this is one of the most important events for science in the past 50 years. Scientists and engineers are coming to realize that the traditional models of a 'scientific' career are outdated. Science careers in the future will be marked by adaptability, entrepreneurialism and self-reliance. It's more than a realignment to a different set of jobs; it's a change in approach to a career. The era of 'career resilience' has begun.

\section{From I-shaped to T-shaped}

When my father embarked on his scientific career, he was advised to choose one topic and aspire to be the best in the world at it. This made great sense. Science was growing in all directions, so even an infinitesimally narrow area of knowledge was bound to expand. The prevailing wisdom was to be an 'I'-shaped scientist, with deep expertise in a single area.

Today, focusing on a single area of science no longer guarantees safety and security. Like an investment portfolio made up of just one stock, careers that are l-shaped are prone to large swings in value. Career experts often recommend that professionals adopt a ' $T$ '-shaped strategy: deep technical expertise is still needed, but capping that depth should be a broad set of interdisciplinary skills that allow a scientist or engineer to solve problems in a wide range of applications. Despite the growth of interdisciplinary centres on some campuses, university training has yet to adapt to this new reality. Traditional PhD programmes still train students to focus on a single area of scholarly research. 'Broadening' activities, such as participation in interdisciplinary studies or taking business or law courses is, at best, tolerated.

\section{How to cap your ' $\mathrm{T}$ '}

Broadening your experience base and your network is now widely recognized to be the most effective way to expand your career options and make your career more resilient.

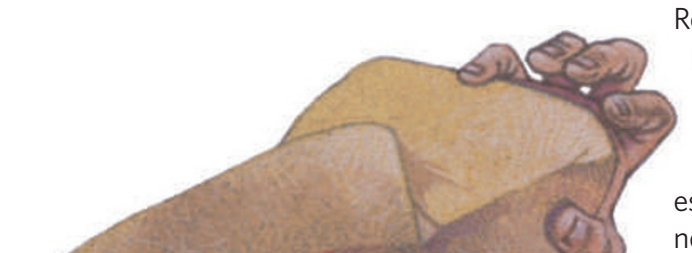
never leave their department. Even short periods spent at another institution, university or company can be enormously broadening, both for your skills and for your network. Exchange assignments as short as four weeks can allow you to meet and interact with numerous new colleagues.

Scientists and engineers tend to be somewhat reserved in building their networks - focusing mainly on whom they know in the research community. In fact, it is the people outside the world of research who may have greatest effect on your career. If you are interested in an area of technology or commerce outside your area of expertise, consider attending a technical meeting on the subject. Interested in the interface of science and business? Attend lectures at the nearest business school. You'd be surprised who you will meet just by mingling.

Career resilience means investing in a range of activities. It means cultivating and maintaining an active network of colleagues, partners, friends and supporters who can steer you towards new opportunities. It means thinking of yourself as an adaptable and clever problem-solver rather than a technical expert with a narrow sliver of knowledge.

Peter Fiske is chief technology officer of PAX Water Technologies in San Rafael, California, and author of Put Your Science to WORK. 